\title{
An evolutionary perspective of the origin of males from unisexual ancestral females
}

\author{
Zhongneng $\mathrm{Xu}^{*}$ \\ Department of Ecology, Jinan University, Guangzhou 510632, China \\ Received July 25, 2016; accepted October 30, 2016; published online December 5, 2016 \\ Citation: $\quad$ Xu, Z. (2017). An evolutionary perspective of the origin of males from unisexual ancestral females. Sci China Life Sci 60, 102-104. doi: 10.1007/s11427- \\ 015-0125-y
}

The urge to understand the cause and evolution of sex both scientifically and philosophically is the result of the development of human civilization and a fascination regarding the existing phenomenon of sexual reproduction. Theories regarding the evolution of sex have been developed by many scientists such as Aristotle, Charles Darwin, August Weismann, Hermann Henking, Nettie Stevens, John Maynard Smith, the Charlesworths, Sarah P. Otto, and A. S. Kondrashov (Maynard Smith, 1978; Kondrashov, 1993; Bachtrog et al., 2014). Many theories tried to provide reasons behind the maintenance of sex, but a sequence in the evolution of sex is needed to formulate different causes for the maintenance during the developing stages. How sex evolved remains a puzzle. Evolutionary relation between meiosis and mitosis, transformation between haploids and diploids, and evolutionary transitions between dioecy and hermaphroditism are contradictory in different explanations. Unisexual reproduction was mostly considered as losing sex (Schön et al., 2009) and originated from hybrids, but genomic sequencing data supported that the bdelloid rotifer Adineta vaga, a parthenogenetic species without males, evolved from an ameiotic ancestor (Flot et al., 2013). In addition, the unequal status of females and males in reproduction and genetic mutations results in a lack of good explanations for how sex evolved. An unknown in parthenogenetic reproduction

*Corresponding author (email: txuzn@jnu.edu.cn) is why females always complete reproduction, but natural males rarely did. The study of sex-biased genes demonstrated higher mutation levels in genes with male-biased expression compared with female-biased or unbiased genes (Ellegren and Parsch, 2007). Therefore, the perspective presented here seeks a sequence in the evolution of sex and tries to provide rational explanations for the link among ploidy, asexual reproduction, parthenogenesis, hermaphroditism, dioecy, the origin of males, and the maintenance of sex.

If the cost of producing a cell is considered, non-meiosis fissions, including mitosis, tended to be selected for reproduction. Most organisms have the potential to develop an individual from a seed cell only via mitosis. The evolution of meiosis is an unsolved complex problem in biology. Wilkins and Holliday reported genetic and cytological evidence to prove the hypothesis that meiosis evolved from mitosis, but why homolog synapsis, a new and key step between mitosis and meiosis, arose is unknown (Wilkins and Holliday, 2009). The present research provides a reason for the emergence of homolog synapsis. The first eukaryote ancestors were haploids and multiplied by mitosis. Diploid species evolved from haploids ones when the chromosomes were copied but the cells did not divide (Figure S1 in Supporting Information). Synapsis is a reunion process of chromosomal copies from an ancestral mother that should have divided into daughter cells long ago. The cross pairing between homologous chromosomes may be one of the forces that induces bivalents. In meiosis, the first phase was not the splitting of sister chromo- 
somes, but the separation of bivalents until the second phase, mitosis. Initially, sex occurred when two meiosis products, called gametes, melted (Figure S1 in Supporting Information). To explain this initial sex and the phenomena in sexual reproduction described later, the term "female" is defined as having reproductive cells that provided nutrients and genetic resources in reproduction. That being the case, female was the first sex, the gametes of which provided both living resources for embryos and genetic information. The original females completed reproduction by themselves. In ancestral females, meiosis and gamete fusion reduced chromosomal errors, conserved genetic molecular sequences, and removed epigenetic changes (Wilkins and Holliday, 2009; Gorelick and Heng, 2011). Of course, more advantageous mutations from the crossover were another reason for organisms to choose the first sex.

The melting of gametes or gamete nuclei was an important reproductive process in ancestral females. Automixis was a method to melt meiosis products within one cell that existed in unicellular organisms. Some mutated meiosis products occurred and enlarged the genetic information from the species when they were melted. The fusion of gametes produced by different cells was an effective way to widely distribute mutations in a population, resulting in the fruitfulness of genetic diversities. Splitting times might be the dominant factor for obtaining mutations, and the more times the cells split, the more mutations the gametes accumulated. Such rapidly mutated gametes were favored over time, and when they were discriminated from female gametes, losing most of the living sources for developing embryos and becoming reduced in size, male gametes came into being (Figure $\mathrm{S} 2$ in Supporting Information). These male gametes co-existed with female gametes in multicellular individuals and were thus called hermaphrodites. If an expanded definition of hermaphrodites was used, one individual by itself producing eggs/egg-producers and sperms/sperm-producers and some parthenogenetic females producing both male and female offspring in parthenogenesis, such as some rotifers and aphids, could be considered as hermaphrodites.

To obtain more genetic diversity, fusion series of meiotic gametes in reproductive evolution occurred within a cell, within an individual, within a species, and between species. The fusion of gametes or nuclei, however, underwent a rejection of the nucleic material and possibly cooperated with other cellular factors from different cells. Apomoxis is the fusion of nucleic materials within the same cell in an acceptable mixture. Other forms of automixis included the melting of offspring cells from the same mother cell, resulting in less rejection. Selfing indicates a mixture of genomes from the same individual, and this process could occur naturally, although it might induce cytologic rejection to some extent. Crossing was more difficult for genetic material compatibility than the cases listed above. There could be a transition from rejection to compatibility in genetic materials. Sex parasitism, rejection of genomes of one sex, is a suitable transition candidate. Another candidate transition was the mechanism of combining genomes from different cells that co-existed as diploids in offspring and had an equal chance to pass on genetic characteristics without chromosomal recombination (Lee et al., 2010).

The origin of males was closely related to cross fertilization. For the gynodioecy species, individual females could mate with hermaphrodites. The benefits of increasing genetic diversity to adapt to environments favored such mating. If this mating occurred with high frequency during the evolutionary period, female organs or female nuclei could gradually degrade in the hermaphrodites. Thus, independent male individuals were developed. In the hermaphroditic species, cross fertilization produced more genetic diversity than self-fertilization. In an evolutionary method of crossing and avoiding self-fertilization in hermaphrodites, female organs and male organs evolved to mature at different times. After an appropriate amount of time and mating among sequential hermaphrodites, males separated from the hermaphrodites and females reoccurred. Some ancestral parthenogenetic species underwent a development strategy, such as a life history, that merged asexual reproduction and dioecism, and male individuals may have directly developed from females, like Marchantia polymorpha and honey bee queens. As mentioned above, whether these parents were hermaphrodites in an expanded definition remains to be discussed. Until the occurrence of males, the evolutionary development of sexes connecting haploids, diploids, hermaphroditism, and dioecy was formed (Figure 1).

Females and males played different roles in sex. The function of females in reproduction was to enable the species to continually survive. Eggs can complete reproduction by themselves through modes such as asexual mitotic reproduction and parthenogenesis without aid from sperms. Male gametes, however, cannot produce embryos by themselves because they contain minimal living resources for embryos. Embryos can be produced with only male genomes but require resources such as plasma, which are provided by other cells, including female gametes. Males produced a larger number of mutations, e.g., there is evidence of a high mutation rate in males and a high divergence of male-biased genes (Li et al., 2002; Ellegren and Parsch, 2007), to provide increased genetic diversity to enable the offspring to adapt to the environments, especially in difficult conditions. However, males are not as important as females for yielding offspring. The lower status of males in reproduction is often observed and includes shorter life spans, frequent unavailability, parasitic characteristics, and being eaten by females after mating.

The main causes of sex evolution might be an opposite effect, reducing genetic mutations in the origin of ancestral fe- 


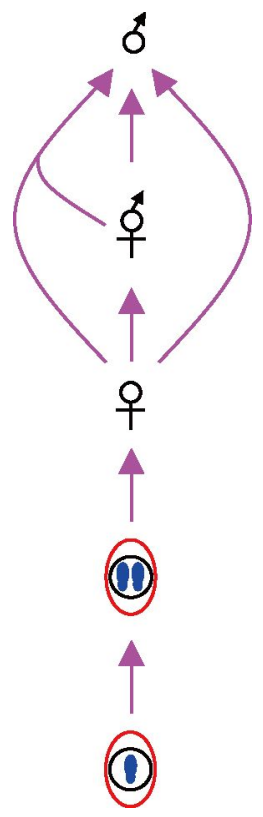

Figure 1 Phylogenetic direction for the occurrence of males. The steps of male origin in the evolution of sex were asexual haploid ancestors, asexual diploid ancestors, females, hermaphrodites, and males. In some cases, the ancestors only had hermaphrodites that evolved from female ancestors, and these hermaphrodite ancestors could develop into females again. Males, in some species, were considered to evolve from hermaphrodites when the female organs degenerated either from hermaphrodites only or from cooperation between females and hermaphrodites. By themselves, parthenogenetic females in some species, such as honey bees and some aphid species, produced haploid or diploid male individuals directly, but whether they were parthenogenetic individuals or hermaphrodites remains unknown.

males but increasing genetic diversity from ancestral females to males. The benefit of two sexes has the trade-off advantages of the conservation of females for the perpetuation of the species and the mutation of males for better genetic diversity. The view that males evolved from ancestral females but were not indispensable could provide new information to the discussion of sex evolution, sex differences, sex competition, gender discrimination, and so on.

\section{Compliance and ethics}

Acknowledgements This work was supported by the United Fund Project of National Natural Science Foundation of China-Guangdong (U1301235).

Bachtrog, D., Mank, J.E., Peichel, C.L., Kirkpatrick, M., Otto, S.P., Ashman, T.L., Hahn, M.W., Kitano, J., Mayrose, I., Ming, R., Perrin, N., Ross, L., Valenzuela, N., Vamosi, J.C., and Vamosi, J.C. (2014). Sex determination: why so many ways of doing it? PLoS Biol 12, e1001899.

Ellegren, H., and Parsch, J. (2007). The evolution of sex-biased genes and sex-biased gene expression. Nat Rev Genet 8, 689-698.

Flot, J.F., Hespeels, B., Li, X., Noel, B., Arkhipova, I., Danchin, E.G.J., Hejnol, A., Henrissat, B., Koszul, R., Aury, J.M., Barbe, V., Barthélémy, R.M., Bast, J., Bazykin, G.A., Chabrol, O., Couloux, A., Da Rocha, M., Da Silva, C., Gladyshev, E., Gouret, P., Hallatschek, O., Hecox-Lea, B., Labadie, K., Lejeune, B., Piskurek, O., Poulain, J., Rodriguez, F., Ryan, J.F., Vakhrusheva, O.A., Wajnberg, E., Wirth, B., Yushenova, I., Kellis, M., Kondrashov, A.S., Mark Welch, D.B., Pontarotti, P., Weissenbach, J., Wincker, P., Jaillon, O., and Van Doninck, K. (2013). Genomic evidence for ameiotic evolution in the bdelloid rotifer Adineta vaga. Nature 500, 453-457.

Gorelick, R., and Heng, H.H.Q. (2011). Sex reduces genetic variation: a multidisciplinary review. Evolution 65, 1088-1098.

Kondrashov, A. S. (1993). Classification of hypotheses on the advantage of amphimixis. J Hered 84, 372-387. .

Lee, S.C., Ni, M., Li, W., Shertz, C., and Heitman, J. (2010). The evolution of sex: a perspective from the fungal kingdom. Microbiol Mol Biol Rev $74,298-340$

Li, W., Yi, S., and Makova, K. (2002). Male-driven evolution. Curr Opin Genet Dev 12, 650-656.

Maynard Smith, J. (1978). The Evolution of Sex. (Cambridge: Cambridge University Press). .

Schön, I., Martens, K., and Dijk, P. (2009). Lost Sex: The Evolutionary Biology of Parthenogenesis. (Berlin: Springer). .

Wilkins, A.S., and Holliday, R. (2009). The evolution of meiosis from mitosis. Genets 181, 3-12.

\section{SUPPORTING INFORMATION}

Figure S1 Origin of the first sex and ways to maintain diploids.

Figure S2 Evolutionary steps from the ancestral female to the hermaphrodite.

The supporting information is available online at life.scichina.com and www.springerlink.com. The supporting materials are published as submitted, without typesetting or editing. The responsibility for scientific accuracy and content remains entirely with the authors. 